\title{
Rehabilitación estética asistida por diseño digital de sonrisa en asimetrías dentofaciales: Reporte de caso.
}

\section{Aesthetic rehabilitation assisted by digital smile design for dentofacial asymmetries: Case report.}

\author{
Jose Manuel Olivares ${ }^{1}$, Nicole Pfeil ${ }^{\star}$, María-Ignacia Sirhan ${ }^{2}$, Alejandro Madariaga ${ }^{1}$
}

\author{
1. Área de Rehabilitación Oral, Pontificia \\ Universidad Católica de Chile, Chile. \\ 2. Estudiante de Pregrado, Pontificia Universidad \\ Católica de Chile, Chile. \\ * Correspondencia Autor: Nicole Pfeil | Dirección: \\ Las Hualtatas Interior 5415, depto 81, Vitacura, \\ Santiago, Chile | Teléfono: +5696249 1755 | \\ E-mail:ndpfeil@uc.cl \\ Trabajo recibido el 29/10/2019. \\ Aprobado para su publicación el 04/02/2020
}

\begin{abstract}
RESUMEN
Tradicionalmente se ha propuesto una coincidencia entre la línea media facial y dentaria como requisito para transmitir una impresión de simetría, balance y armonía estética. Sin embargo, esta situación puede ser compleja de lograr en rostros asimétricos, impulsando el desarrollo de nuevos protocolos de análisis dentofacial para obtener resultados armónicos y conservadores integrando las estructuras anatómicas que se desvíen de las normas establecidas. Caso: El objetivo de este reporte de caso es presentar una rehabilitación estética integral en base a prótesis fija unitaria libre de metal, asistida por diseño digital de sonrisa, en un paciente con asimetría facial utilizando el concepto de flujo facial (FF) como protocolo de análisis estético dentofacial. Conclusiones: El concepto de FF establece parámetros estéticos dentro de rangos de armonía, permitiendo planificar rehabilitaciones más conservadoras. Sin embargo, faltan estudios para determinar el impacto de este análisis.
\end{abstract}

PALABRAS CLAVE:

Estética dental; Flujo facial; Análisis dentofacial; Línea media.

Int. J. Inter. Dent Vol. 13(2); 102-104, 2020.

\section{ABSTRACT}

The traditional aesthetic parameters demand a coincidence between the facial and dental midlines that transmits an impression of symmetry, balance and harmony. However, the complexity of achieving this standard in faces with large asymmetries, has driven to the development of new dentofacial analysis protocols such as the concept of facial flow (FF), in order to obtain harmonic and conservative results integrating the structures that deviate from the norm. Case: The aim of this case report is to present an integral aesthetic rehabilitation based on metal-free fixed prostheses assisted by digital smile design in a patient with facial asymmetry using the concept of FF. Conclusions: The use of dentofacial aesthetic analysis protocols such as FF aids in the development of harmonic and less invasive dental treatments. However, due to the recent appearance of the FF concept, further studies are needed to reveal the implications of this analysis. KEY WORDS:

Dental aesthetic; Facial flow; Dentofacial analysis; Midline.

Int. J. Inter. Dent Vol. 13(2); 102-104, 2020.

\section{INTRODUCCIÓN}

En la actualidad predomina el concepto de armonía dentofacial como el estándar de belleza mundial, requiriendo una simetría, proporción, color e integración de la sonrisa con las características faciales individuales. Variables como diastemas, discromías, malposiciones dentarias y maloclusiones anatómicas pueden afectar considerablemente la apreciación estética ${ }^{(1)}$. Para la evaluación estética dentofacial se han desarrollado progresivamente nuevas herramientas de análisis y planificación, como el diseño digital de sonrisa (DSD) ${ }^{(2)}$, que permiten previsualizar los resultados deseados; integrando al equipo clínico, al laboratorio y al paciente en la toma de decisiones. De esta manera, se busca dar una respuesta eficiente a las necesidades estéticas crecientes de la población entendiendo el impacto psicológico y social de este tema.

Dentro de los parámetros más relevantes del análisis estético dentofacial se encuentra el componente vertical, establecido por la coincidencia de la posición relativa de la línea media dentaria (LMD) respecto a la línea media facial (FM). Esta norma es controversial, existiendo distintas definiciones de FM que coinciden en una línea perpendicular a los planos horizontales de referencia (bipupilar, intercomisural), sin embargo, difieren en su posición final impactando directamente en la planificación y ejecución de tratamientos odontológicos $^{(3)}$. (Tabla 1) Si bien la coincidencia entre LMD y FM es deseable ya que transmite una impresión de simetría, balance y armonía, existe un rango de tolerancia ${ }^{(3)}$. Según Pereira y cols, una diferencia de hasta $4 \mathrm{~mm}$ es imperceptible siempre que se mantenga un paralelismo entre ambas líneas ${ }^{(4)}$

En el caso de inclinaciones, un leve canteo, de tan solo $3.5^{\circ}$, puede afectar negativamente la estética facial(5). Silva y cols ${ }^{(3)}$, publicaron el 2018 el concepto de flujo facial (FF) para la evaluación estética vertical, entendiendo que los rostros son por naturaleza asimétricos y, que por tanto, no pueden ser analizados únicamente con un trazado de líneas rectas. Mediante la unión de diferentes puntos faciales de referencia, se define una tendencia de la trayectoria de las estructuras faciales centrales (nariz, labios, mentón), determinando la posibilidad de lograr una inclinación armónica de la LMD hacia el mismo lado de la desviación facial. De esta manera, se propone un enfoque orgánico y conservador, integrando las asimetría faciales dentro de la planificación estética. 
El objetivo de este reporte de caso consiste en presentar una rehabilitación estética integral en base a prótesis fija unitaria (PFU) libre de metal, asistida por DSD, en un paciente con asimetría facial utilizando el concepto de FF. Además, se enunciará la secuencia de tratamiento detallando los materiales y técnicas utilizadas.

Tabla 1: Controversia respecto a definiciones de LM.

\begin{tabular}{l|l}
\multicolumn{1}{c|}{$\begin{array}{c}\text { Autores } \\
\text { (Año) }\end{array}$} & \multicolumn{1}{c}{ Definición de Línea Media Facial } \\
\hline $\begin{array}{l}\text { Owens et al } \\
(\mathbf{2 0 0 2 )})^{(11)}\end{array}$ & Línea bisectriz a la distancia interpupilar. \\
\hline $\begin{array}{l}\text { Bidra and Cols } \\
(\mathbf{2 0 0 2 )}\end{array}$ & $\begin{array}{l}\text { Línea bisectriz a la distancia entre cantos } \\
\text { externos de los ojos. }\end{array}$ \\
\hline $\begin{array}{l}\text { Miller and Jamison } \\
(\mathbf{1 9 7 4})^{(13)}\end{array}$ & $\begin{array}{l}\text { Consideran el filtrum como el punto más } \\
\text { confiable para definir la línea media. }\end{array}$ \\
\hline
\end{tabular}

\section{REPORTE DEL CASO}

Paciente sexo femenino, 58 años, sin antecedentes sistémicos, hábito dietético con alto contenido ácido y alto riesgo cariogénico según análisis CAMBRA $^{(6)}$. Consulta por la deficiente estética de sus dientes y para la resolución de posibles problemas orales.

Se realiza la evaluación clínica y radiográfica detectando: desdentamiento parcial bimaxilar con ausencia de dientes 2.5 y 4.1, maloclusión anatómica con migraciones dentarias múltiples, apiñamiento del sector anteroinferior, mordida cruzada entre dientes 2.3 y 3.3 y maloclusión funcional, con interferencias en las guías excursivas ${ }^{(7)}$. Además, se observa enfermedad periodontal activa con diagnóstico de periodontitis estadío I generalizada grado $\mathrm{B}^{(8)}$, múltiples dientes con patologías endodónticas como necrosis pulpar en $1.1,2.1$ y 2.2 y periodontitis apical asintomática en diente previamente tratado en 1.2 y 2.4 ; lesiones de caries activas y restauraciones deficientes en estado Charlie de acuerdo a los criterios Ryge-USPHS en el sector anterosuperior ${ }^{(9)}$. Previo al tratamiento estético, se resuelven las patologías anteriormente mencionadas hasta lograr un estado compatible con salud.

La primera etapa consistió en el análisis dentofacial clínico y digital (DSD) donde se detectó: asimetría facial con inclinación de la nariz, filtrum y mentón hacia la derecha, incisivos centrales superiores triangulares y desproporcionados, balance gingival alterado, corredor bucal asimétrico, línea labial alta, curva de la sonrisa cóncava y desviación e inclinación de la LMD respecto a la FM de $2 \mathrm{~mm}$ y $5^{\circ}$ respectivamente. Para evaluar la coincidencia entre ambas líneas utilizando el DSD, se realiza un trazado perpendicular y equidistante a la línea bipupilar (LB) (Figura 1), determinando, en este caso, una propuesta de tratamiento invasiva, requiriendo la intervención de todo el sector anterosuperior y primeros premolares para su corrección. Por lo tanto, debido a la asimetría facial del paciente, se decidió realizar un segundo análisis dentofacial considerando un enfoque más conservador y orgánico. Esto, se logra mediante la incorporación del FF con el objetivo de evaluar la integración de la LMD a los otros componentes faciales determinando la rehabilitación final(3).

Silva y $\operatorname{cols}^{(3)}$ proponen la división del rostro en dos áreas, una verde determinada por la dirección del FF y otra roja correspondiente a la opuesta. Cualquier desviación o inclinación leve que se encuentre dentro del área verde, será menos perceptible, disminuyendo la tensión visual al

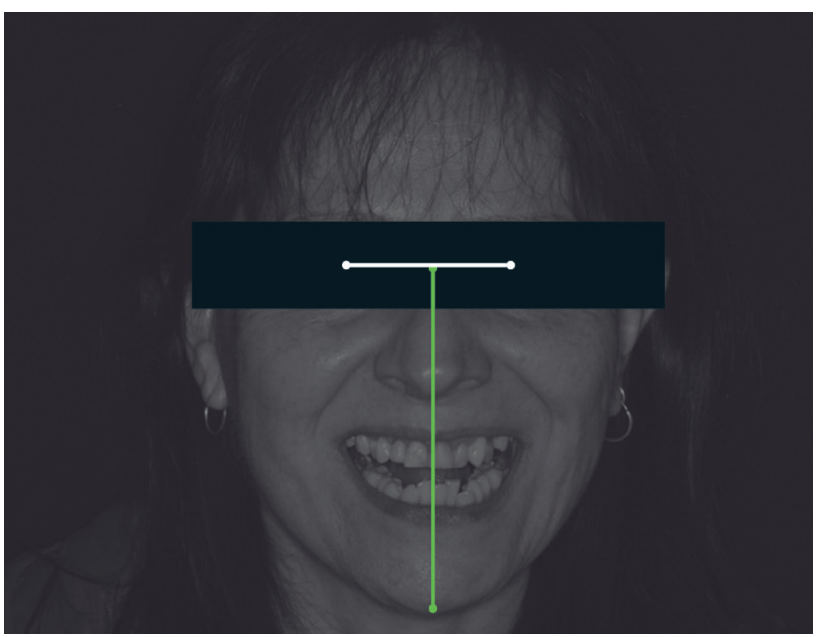

Figura 1. Trazado de línea media facial perpendicular a línea bipupilar. incorporarse al flujo de los demás componentes faciales. En este caso, el área verde corresponde al lado derecho y asimismo la desviación natural de su LMD respecto a la FM. (Figura 2) Por lo tanto, se decidió rehabilitar el sector anterosuperior manteniendo la desviación e inclinación inicial de la LMD, logrando un tratamiento más conservador. (Figura 3)

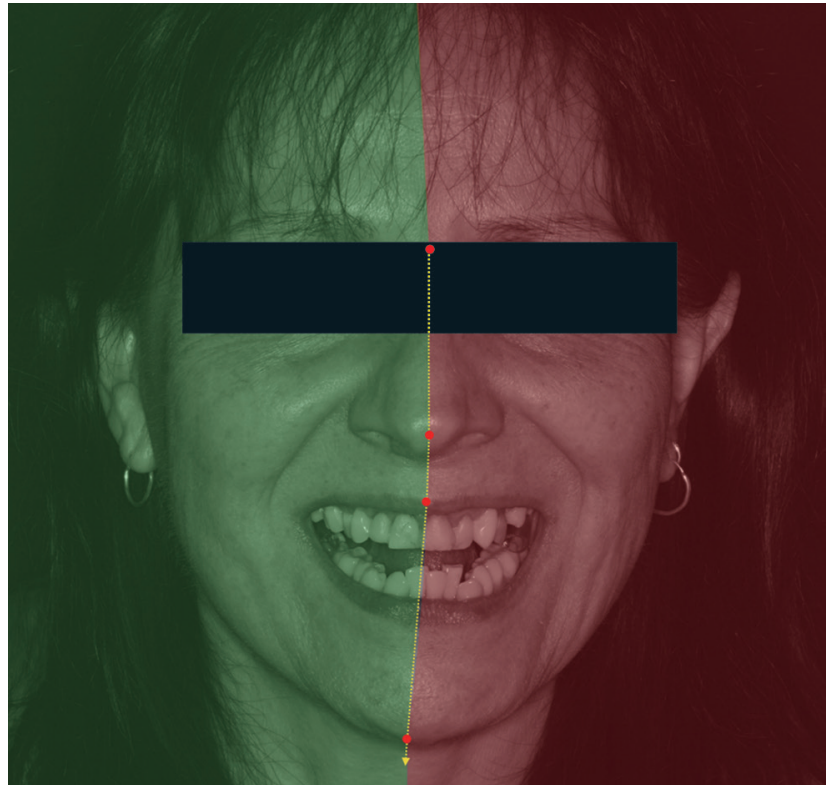

Figura 2. Movimiento direccional del flujo facial hacia el lado derecho o "área verde".

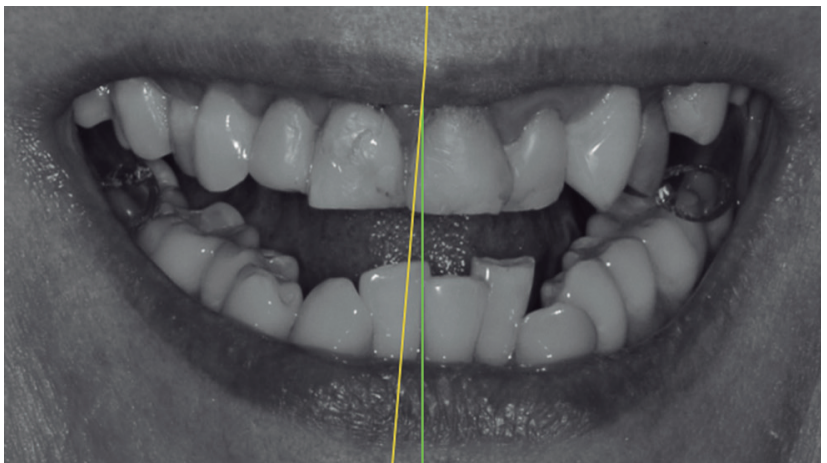

Figura 3. Acercamiento de sonrisa, comparando LM (verde) y FF.

Según el segundo DSD modificado en base al FF, se confeccionó un encerado diagnóstico de los dientes anterosuperiores nivelando los cénit gingivales y corrigiendo la asimetría coronaria inicial con proporciones de $100 \%$ y $120 \%$ en dientes 1.1 y 2.1 respectivamente, determinando una proporción final ideal de $80 \%$ en ambos. Ante la negativa del paciente a someterse a un procedimiento quirúrgico para corregir la desarmonía en su balance gingival, se decide en forma conjunta modificar únicamente las proporciones coronarias. Posteriormente, se procedió a realizar el tallado de las preparaciones biológicas para PFU de los incisivos anterosuperiores y su provisionalización. (Figura 4).

Considerando la alta demanda estética del caso y con el fin de lograr un color armónico en relación a los dientes adyacentes, se optó por PFU libre de metal con núcleo de zirconio y cerámica de revestimiento logrando opacar el sustrato dentario cromáticamente alterado. Además, según la dificultad de aislación en relación con la ubicación intrasulcular de la línea de terminación cervical, se prefirió una cementación convencional con cemento de vidrio ionómero. Finalmente, se obteniene como resultado final PFUs libres de metal integradas exitosamente con los dientes vecinos y antagonistas logrando reestablecer la estética y funcionalidad de la guía anterior. (Figura 5)

\section{DISCUSIÓN}

Actualmente existe una creciente demanda de tratamientos odontológicos estéticos. Esto, se condice con el gran impacto psicológico que genera una sonrisa, donde no solo determina un atractivo físico, si no que también influencia la percepción de un tercero acerca de la inteligencia, personalidad 


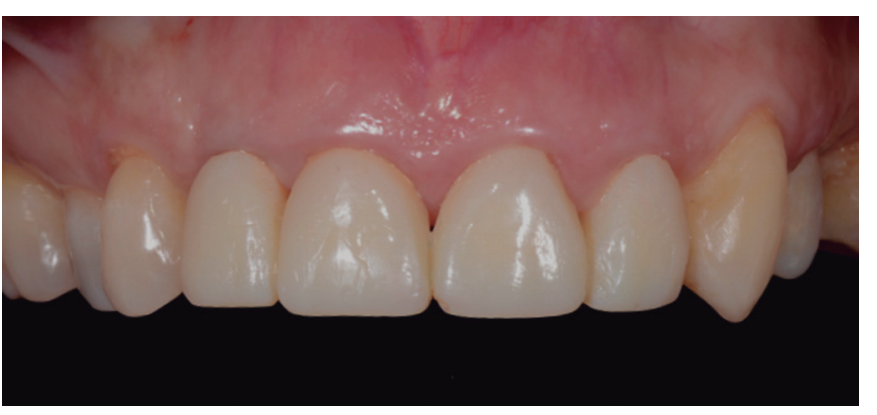

Figura 4. Provisionales de BisAcryl como propuesta rehabilitadora estética.

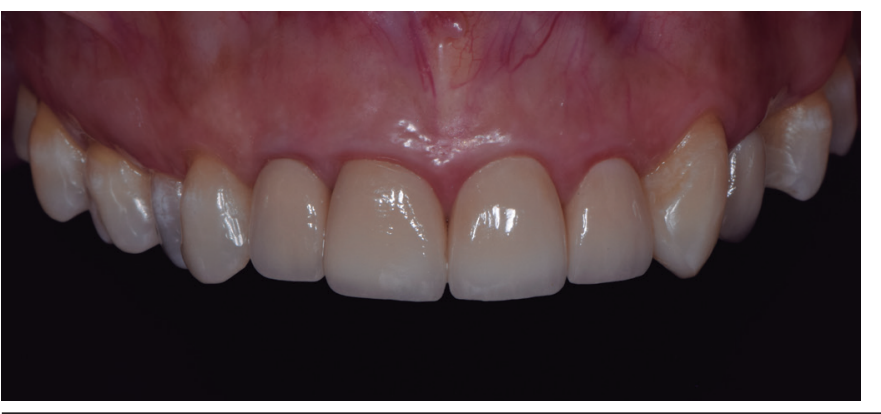

Figura 5. Resultado final inmediato post-cementación.

y comportamiento social de un individuo ${ }^{(10,11)}$.

La determinación de la FM es un aspecto crítico en el análisis estético dentofacial. Debido a que la forma asimétrica de los rostros humanos imposibilita la existencia de criterio objetivo único para su evaluación, existe controversia respecto a los métodos para su ubicación ${ }^{(12,13,14,15)}$ (Tabla 1) Para cumplir con las normas tradicionales y lograr la coincidencia entre la FM y la LMD ${ }^{(14)}$, puede ser incluso necesario realizar tratamientos complejos o invasivos, sobretodo en pacientes con grandes asimetrías dentofaciales. Realizar un análisis en base al FF permite que las rehabilitaciones se mimeticen con la apariencia física de cada paciente, siguiendo la tensión de sus diferentes estructuras faciales e integrando canteos y/o desviaciones de la LMD de manera conservadora e individualizada. (Figura 6)

\section{CONCLUSIÓN}

La selección de análisis estéticos como el concepto de FF, permite integrar

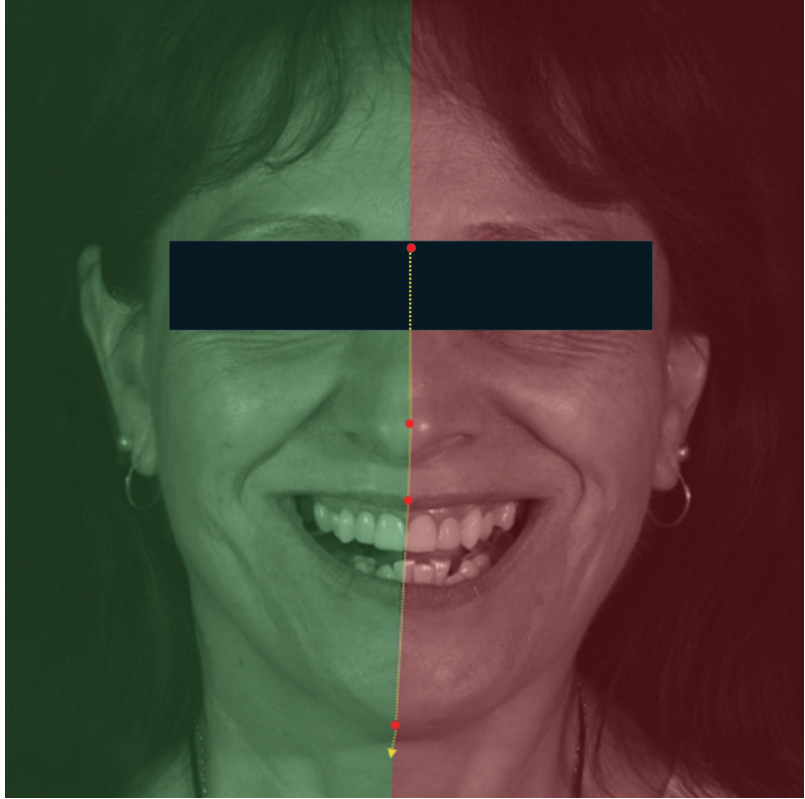

Figura 6. Rehabilitación final según movimiento direccional del flujo facial.

los parámetros dentofaciales dentro de un rango de armonía que permite realizar rehabilitaciones más conservadoras que con métodos tradicionales. Sin embargo, faltan estudios para determinar el impacto de este análisis en la percepción social y autopercepción estética de los pacientes.

\section{RELEVANCIA CLÍNICA}

Este reporte de caso presenta una manera menos invasiva de rehabiltar el sector anterior manteniendo la desviación de la línea media facial logrando resultados estéticos y armónicos.

\section{CONFLICTO DE INTERÉS}

Los autores declaran no tener conflictos de interés.

\section{FUENTE DE FINANCIAMIENTO}

Este trabajo no cuenta con financiamiento alguno.

\section{Bibliografía}

1. De Deus C, Rodrigues T, Magnani R, Salete M, Machado C, Osmir, et al. The perception of smile attractiveness variations from esthetic norms, photographic framing and order of presentation. Angle Orthod [edición electrónica]. 2009 [citado 25 abril 2019];79(4):634. Disponible en: https://www.angle.org/doi/ pdf/10.2319/030508-131.1

2. Coachman C, Van Doreen E, Gürel G, Lansberg C, Calamita M, Bichacho N. Smile design: From digital treatment planning to clinical reality. Interdiscip Treat Plan [edición electrónica]. 2012 [citado 27 abril 2019];2:119-74. Disponible en: https://go.digitalsmiledesign.com/hubfs/DSD $\% 20$ Articles/Articles $\% 20$ by $\% 20$ CC\%20and\%20Team/Smile\%20Design\%20From\%20Digital\%20Treatment $\% 20$ Planning\%20to\%20Clinical\%20Reality.pdf

3. Silva BP, Mahn E, Stanley K, Coachman C. The facial flow concept: An organic orofacial analysis-the vertical component. J Prosthet Dent. [edición electrónica]. 2019 [citado 28 marzo 2019];121(2):189-94. Disponible en: https://linkinghub. elsevier.com/ retrieve/pii/S0022391318302750

4. V O Kokich Jr, H A Kiyak, P A Shapiro Comparing the perception of dentists and lay people to altered dental esthetics. J Esthet Dent. [edición electrónica]. 1999 [citado 28 marzo 2019];11(6):311-24. Disponible en:https://onlinelibrary.wiley.com/ doi/abs/10.1111/j.1708-8240.1999.tb00414.x

5. Silva BP, Jiménez-Castellanos E, Stanley K, Mahn E, Coachman C, Finkel S. Layperson's perception of axial midline angulation in asymmetric faces. J Esthet Restor Dent. [edición electrónica]. 2018 [citado 28 marzo 2019];30(2):119-25. Disponible en: https://onlinelibrary.wiley.com/doi/abs/10.1111/j.1708-8240.1999. tb00414.x

6. Featherstone JDB, Adair SM, Anderson MH, Berkowitz RJ, Bird WF, Crall JJ, et al. Caries management by risk assessment: consensus statement, April 2002. J Calif Dent Assoc. [edición electrónica]. 2003 [citado 28 marzo 2019] ;31(3):257-69. Disponible en: https://europepmc.org/article/med/12693825

7. Manns Freese AE. Manual práctico de oclusión dentaria. 2a ed. México Editor Amolca. 2006.

8. Caton JG, Armitage G, Berglundh T, Chapple IL, Jepsen S, Kornman KS, et al. A new classification scheme for periodontal and peri-implant diseases and conditions-
Introduction and key changes from the 1999 classification. J Clin Periodontol. [edición electrónica]. 2018 [citado 6 junio 2019];45:45. Disponible en: https://www. fippdentalearning.org/fiip/wp-content/uploads/2018/06/New-Classification-forPeriodontal-Diseases-2017.pdf

9. Ryge G, Snyder M. Evaluating the clinical quality of restorations. J Am Dent Assoc. [edición electrónica].1973 [citado 16 mayo 2019];87(2):369-77. Disponible en: https://jada.ada.org/article/S0002-8177(73)72055-9/pdf

10. Olsen JA, Inglehart MR. Malocclusions and perceptions of attractiveness intelligence, and personality, and behavioral intentions. Am J Orthod Dentofac Orthop. [edición electrónica. 2011 [citado 16 mayo 2019];140(5):669-79. Disponible en: https://linkinghub.elsevier.com/retrieve/pii/S0889540611006780

11. Van der Geld P, Oosterveld P, Van Heck G, Kuijpers-Jagtman AM. Smile Attractiveness. Angle Orthod. [edición electrónica]. 2007 [citado 6 junio 2019];77(5):75965. Disponible en: https://www.researchgate.net/publication/6153330_Smile_ attractiveness_Self-perception_and_influence_on_personality

12. Owens EG, Goodacre CJ, Loh PL, Hanke Ğ, Okamura M, Jo K, et al. A multicenter interracial study of facial appearance. Part 1: A comparison of extraoral parameters. Int J Prosthodont. [edición electrónica]. 2002 [citado 27 mayo 2019];15(3):273-82. Disponible en: https://pubmed.ncbi.nlm.nih.gov/12066491/

13. Bidra AS, Uribe F, Taylor TD, Agar JR, Rungruanganunt P, Neace WP. The relationship of facial anatomic landmarks with midlines of the face and mouth. J Prosthet Dent. [edición electrónica] 2009 [citado 6 junio 2019];102(2):94-103. Disponible en: https://pdfs.semanticscholar.org/4a52/170d2f4dacb76d719df2aa3e b808fcc7210e.pdf

14. Miller EL, Bodden WR, Jamison HC. A study of the relationship of the dental midline to the facial median line. J Prosthet Dent. [edición electrónica]. 1979. [citado 27 mayo 2019];41(6):657-60. Disponible en: https://europepmc.org/article/ $\mathrm{med} / 374721$

15. Cardash HS, Ormanier Z, Laufer B-Z. Observable deviation of the facial and anterior tooth midlines. J Prosthet Dent. [edición electrónica]. 2003. [citado 27 mayo 2019];89(3):282-5. Disponible en: https://www.sciencedirect.com/science/article/pii/ S0022391302527682 